\title{
Stabilization of the electroweak scale in 3-3-1 models
}

\author{
Alex G. Dias ${ }^{1}$ and V. Pleitez ${ }^{2}$ \\ ${ }^{1}$ Centro de Ciências Naturais e Humanas, Universidade Federal do ABC, R. Santa Adélia 166, Santo André-SP, 09210-170, Brazil \\ ${ }^{2}$ Instituto de Física Teórica, UNESP-São Paulo State University, Caixa Postal 70532-2, São Paulo-SP, 01140-070, Brazil
}

(Received 14 August 2009; published 28 September 2009)

\begin{abstract}
One way of avoiding the destabilization of the electroweak scale through a strong coupled regime naturally occurs in models with a Landau-like pole at the TeV scale. Hence, the quadratic divergence contributions to the scalar masses are not considered as a problem anymore since a new nonperturbative dynamic emerges at the $\mathrm{TeV}$ scale. This scale should be an intrinsic feature of the models, and there is no need to invoke any other sort of protection for the electroweak scale. In some models based on the $S U(3)_{C} \otimes S U(3)_{W} \otimes U(1)_{X}$ gauge symmetry, a nonperturbative dynamics arise and it stabilizes the electroweak scale.
\end{abstract}

DOI: 10.1103/PhysRevD.80.056007

PACS numbers: 11.15.Ex, 12.60.Cn

Understanding the standard model (SM) of strong and electroweak interactions as an effective description of a more fundamental theory implies that, at an energy scale denoted by $\Lambda$, new physics must arise. Without any additional hypotheses, it is natural that $v_{W}<\Lambda \leq M_{\mathrm{Pl}}$, where $v_{W}=\left(\sqrt{2} G_{F} / 2\right)^{1 / 2} \simeq 246 \mathrm{GeV}$ is the electroweak scale and $M_{\mathrm{Pl}} \sim 10^{19} \mathrm{GeV}$ is the Planck scale, which is linked to the gravitational interactions according to semiclassical arguments. However, since there is no symmetry at the quantum level protecting the tree-level SM Higgs particle squared mass, $M_{H}^{2}=-2 \mu^{2}$, it receives quantum corrections so that $m_{H}^{2} \rightarrow \bar{M}_{H}^{2}=-2\left(\mu^{2}+\delta \mu^{2}\right)=2(\lambda+$ $\delta \lambda) v_{W}^{2}$. Here $\mu^{2}$ and $\lambda$ are the usual tree-level parameters of the quadratic and the quartic terms in the renormalizable scalar potential at tree level, $V_{0}=\mu^{2} H^{\dagger} H+\lambda\left(H^{\dagger} H\right)^{2}$, with $H$ the usual scalar doublet of the standard model; $\delta \mu^{2}$ and $\delta \lambda$ stand for the corresponding quantum corrections to the tree-level parameters. It so happens that $\delta \mu^{2}$ is proportional to $\Lambda^{2}$, and $\delta \lambda$ is proportional to $\ln \Lambda$ [1]. Hence, the electroweak scale does not seem to be stable against quantum corrections unless some mechanism protects it. In the context of the SM, the Higgs, $Z^{0}$ and $W$ bosons, and the top quark give the dominant contributions for the one-loop effective potential. Taking into account that $\Lambda$ is the cutoff appearing in the momentum integrals, we have, with $g, g^{\prime}$, and $y_{T}$, the $S U(2), U(1)_{Y}$, and the top quark coupling constants of the SM, respectively,

$$
\begin{aligned}
\delta \mu^{2} & \approx 3\left[2 M_{W}^{2}+M_{Z}^{2}+M_{H}^{2}-4 M_{T}^{2}\right]\left(\frac{\Lambda}{4 \pi v_{W}}\right)^{2} \\
& =3\left[\frac{3}{4} g^{2}+\frac{1}{4} g^{\prime 2}+2 \lambda-2 y_{T}^{2}\right]\left(\frac{\Lambda}{4 \pi}\right)^{2},
\end{aligned}
$$

plus terms proportional to $\ln \Lambda$ times loop factors. Independently of the value of $\Lambda$, a Higgs boson with a tree-level mass $M_{H} \approx 310 \mathrm{GeV}$ (i.e. $\lambda \approx 0.8$ ) could make $\delta \mu^{2}$ in Eq. (1) near zero [2]. However, according to the electroweak precision data, such a heavy Higgs seems not to be favored by the global fit of the SM $[3,4]$.

Ideas for stabilizing the electroweak scale have been put forward in the past. We assume that supersymmetry (SUSY) at the TeV scale was probably the first of such ideas[5]. More recently, solutions such as the little Higgs (LH) [6,7] and extra dimension (ED) models [8] were suggested. The LH models are constructions where the $\mathrm{SM}$ is contained in the low energy spectrum with the Higgs boson as a pseudo-Nambu-Goldstone boson of some particular global symmetry. This global symmetry protects one-loop quadratic divergences in $\delta \mu^{2}$. In LH models the common point resides in the fact that they are nonrenormalizable, defining, in principle, an energy cutoff which is around $\Lambda=10 \mathrm{TeV}$ for the internal momentum integrals. It is interesting that this mechanism can be implemented if global and local $S U(3)$ symmetries are considered. In fact, Ref. [9] worked out a LH model which includes right-handed neutrinos transforming nontrivially under $S U(3)_{W} \otimes U(1)_{X}$ gauge symmetry. This was considered previously as an extension of the electroweak interactions in Ref. [10].

Notwithstanding, there are other motivations for considering $S U(3)_{W} \otimes U(1)_{X}$ symmetry as being realized at the $\mathrm{TeV}$ scale, as in the so-called 3-3-1 models [10-12]. One important point is that in some of these models [11,12], depending on the representation content, there is an intrinsic cutoff: the $U(1)_{X}$ coupling constant gets a Landau-like pole of the order of a few TeV [13-16]. Thus, the theory becomes strongly coupled at the $\mathrm{TeV}$ scale inducing, in this way, a natural cutoff for the quantum corrections.

One of the reasons that $\Lambda$ can be arbitrarily high in the $\mathrm{SM}$ is that none of its gauge coupling constants reaches a value which invalidates the perturbative expansion at a testable energy. Let us imagine that a new particle content, which arises from a symmetry structure already revealed at the $\mathrm{TeV}$ scale, drives the gauge coupling constants towards 
a region of values where the theory is nonperturbative. This would indicate that the theory goes to a strongly coupled regime for higher energies. That is the case if the electroweak sector of the SM is embedded into an $S U(3)_{W} \otimes$ $U(1)_{X}$ symmetry, as in a specific class of 3-3-1 models $[11,12]$. Their symmetry reduction, $S U(3)_{W} \otimes U(1)_{X} \rightarrow$ $S U(2)_{W} \otimes U(1)_{Y}$, to the SM group is realized through a scalar field condensation by a vacuum expectation value $\left\langle\chi^{0}\right\rangle=v_{\chi}$, related to the energy scale denoted $\mu_{331}$, by $\sqrt{2} v_{\chi} \approx \mu_{331}$. Below $\mu_{331}$ we have an effective $S U(2)_{W} \otimes$ $U(1)_{Y}$ symmetry with the SM fermions, gauge boson multiplets, and two scalar doublets composing the light degrees of freedom. These are the active degrees of freedom below $\mu_{331}$. All other fields are presumed to be heavy, i.e., with masses around $\mu_{331}$.

In these 3-3-1 models there is the relation

$$
\alpha_{X}\left(\mu_{331}\right)=\frac{\alpha\left(\mu_{331}\right)}{1-4 \sin ^{2} \theta_{W}\left(\mu_{331}\right)},
$$

where $\alpha_{X}=g_{X}^{2} / 4 \pi$ is the gauge coupling constant of the $U(1)_{X}$ gauge factor, written in terms of the electroweak mixing angle $\theta_{W}$, and the electromagnetic coupling $\alpha$, both defined at the $\mu_{331}$ scale. This relation is used to determine the initial value of $\alpha_{X}\left(\mu_{331}\right)$, making the evolution of $\sin ^{2} \theta_{W} \equiv \sin ^{2} \theta_{W}\left(M_{Z}\right) \approx 0.231$ and $\alpha \equiv \alpha\left(M_{Z}\right) \approx 1 / 128$ from the $Z^{0}$ pole to the $\mu_{331}$ scale. We see that a Landaulike pole will be developed as $\sin ^{2} \theta_{W}(\mu)$ evolves to the value 0.25 . This value is reached for $\mu \approx 4.2 \mathrm{TeV}$, making the evolution only with the active degrees of freedom below $\mu_{331}$. This means that even if $\mu_{331}$ is above this value, the cutoff in Eq. (1) must be such that $\Lambda<4.2 \mathrm{TeV}$. Before reaching this pole, $\alpha_{X}$ goes outside the perturbative regime, and we cannot draw any conclusion based on perturbation theory. From the running equation for $\alpha_{X}$, with the initial point as in Eq. (2), the upper energy limit $\bar{\Lambda}<\Lambda$ where the perturbative treatment loses its validity, i.e., $\alpha_{X}(\bar{\Lambda}) \approx 1$, is

$$
\bar{\Lambda}=\mu_{331}\left(\frac{M_{Z}}{\mu_{331}}\right)^{7 / 13} e^{(\pi / 13)\left[(1 / \alpha)\left[1-4 \sin ^{2} \theta_{W}\right]-1\right]} .
$$

The model predicts a mass relation between the neutral $Z^{\prime}$ and the double charged $M_{U}$ gauge bosons,

$$
\frac{M_{Z^{\prime}}^{2}}{M_{U}^{2}} \approx \frac{4 \cos ^{2} \theta_{W}}{3-12 \sin ^{2} \theta_{W}},
$$

with $M_{U}^{2} \approx g^{2} \mu_{331}^{2} / 8$. So, using the lower bound for the $Z^{\prime}$ mass obtained in Ref. [17] as $M_{Z^{\prime}} \geq 620 \mathrm{GeV}$, which implies the minimal value $\mu_{331}^{\min } \approx 750 \mathrm{GeV}$ in Eq. (3), we have $\bar{\Lambda} \approx 2 \mathrm{TeV}$. On the other hand, using the lower bound of $M_{U} \geq 750 \mathrm{GeV}$ [18] for the mass of the double charged gauge boson in the model, $\mu_{331}^{\min } \approx 3.2 \mathrm{TeV}$ so that $\bar{\Lambda}=4 \mathrm{TeV}$. It was pointed out in Ref. [19] that even for $M_{Z^{\prime}} \simeq 1.4$, thousands of new single charged vector bosons presented in the model could be produced at the LHC, pointing out the possibility of distinguishing this model from other models. For other aspects of gauge boson phenomenology, see also [20].

Here we will use the effective potential $[21,22]$ in the formalism of Ref. [23]. Since $\Lambda$ is an upper limit for evaluating the integrals, omitting constant terms proportional to $\Lambda^{4}$, the one-loop contribution to the effective potential is

$$
\begin{aligned}
V_{1}(h) \approx & \frac{1}{64 \pi^{2}} \sum_{i} n_{i}\left[2 \Lambda^{2} \operatorname{Tr}\left(\mathbf{M}_{\mathbf{i}}^{\dagger} \mathbf{M}_{\mathbf{i}}\right)\right. \\
& \left.+\operatorname{Tr}\left\{\left(\mathbf{M}_{\mathbf{i}}^{\dagger} \mathbf{M}_{\mathbf{i}}\right)^{2}\left(\ln \frac{\mathbf{M}_{\mathbf{i}}^{\dagger} \mathbf{M}_{\mathbf{i}}}{\Lambda^{2}}-\frac{1}{2}\right)\right\}\right],
\end{aligned}
$$

where $n_{i}$ is the number of degrees of freedom of the field $i$, including a minus sign for fermions; $\mathbf{M}_{\mathbf{i}} \equiv \mathbf{M}_{\mathbf{i}}(h)$ are obtained from the tree-level mass matrices of the model using $v_{W}=h$. We will consider below only terms proportional to $\Lambda^{2}$, since they are the most relevant contributions for our purposes.

The model we consider has an approximate global $S U(3)_{L} \otimes S U(3)_{R}$ symmetry [24]. In the scalar sector this global symmetry is supposed to be exact. Defining the tritriplet $\Phi=(\eta \rho \chi)$ transforming as $\Phi \rightarrow \Omega_{L} \Phi \Omega_{R}^{\dagger}$ under $S U(3)_{L} \otimes S U(3)_{R}$, the scalar potential is

$$
\begin{aligned}
V[\Phi]= & \mu^{2} \operatorname{Tr}\left(\Phi^{\dagger} \Phi\right)+\lambda_{1}\left[\operatorname{Tr}\left(\Phi^{\dagger} \Phi\right)\right]^{2}+\lambda_{2} \operatorname{Tr}\left(\Phi^{\dagger} \Phi\right)^{2} \\
& +\frac{f}{6} \epsilon_{i j k} \epsilon_{m n l}\left(\Phi_{i m} \Phi_{j n} \Phi_{k l}+\text { H.c. }\right) .
\end{aligned}
$$

The Yukawa interactions and the gauge interactions introduced by $U(1)_{X}$ symmetry explicitly break $S U(3)_{L} \otimes$ $S U(3)_{R}$. For the Yukawa Lagrangian we have

$$
\begin{aligned}
-\mathcal{L}_{Y}= & \bar{Q}_{i L}\left[g_{i \alpha}^{u} u_{\alpha R} \rho^{*}+g_{i \alpha}^{d} d_{\alpha R} \eta^{*}+g_{i k}^{j} j_{k R} \chi^{*}\right] \\
& +\bar{Q}_{3 L}\left[y_{\alpha}^{u} u_{\alpha R} \eta+y_{\alpha}^{d} d_{\alpha R} \rho+y^{J} J_{R} \chi\right] \\
& +\bar{\Psi}_{a L}\left[g_{a b}^{\nu} \nu_{b R} \eta^{*}+g_{a b}^{l} l_{b R} \rho^{*}+g_{a b}^{E} E_{b R} \chi^{*}\right] \\
& + \text { H.c., }
\end{aligned}
$$

where repeated indices are to be summed accordingly with the fields. Terms like $y_{a b} \epsilon_{i j k} \bar{\Psi}_{i a L}\left(\Psi_{j b L}\right)^{c} \eta_{k}$ and $m_{a b} \nu_{a R}\left(\nu_{b R}\right)^{c}$ are not relevant for us here. We assume these terms are forbidden by some symmetry. The global and local symmetries are spontaneously broken with the vacuum expectation value for $\Phi$,

$$
\langle\Phi\rangle=\frac{1}{\sqrt{2}} \operatorname{diag}\left(v_{\eta}, \quad v_{\rho}, \quad v_{\chi}\right),
$$

leaving only the electromagnetic $U(1)$ factor as local symmetry.

In the Appendix the tree-level $\mathbf{M}_{\mathbf{i}}\left(v_{\eta}, v_{\rho}, v_{\chi}\right)$ matrices needed to obtain the quadratic corrections for the present model are shown. Taking the first term in Eq. (5) and the trace of the matrices in the Appendix, we get the one-loop corrections for the bilinears in the potential 


$$
\begin{aligned}
V_{\mathrm{eff}}= & \left(\mu^{2}+\delta \mu_{\eta}^{2}\right) \eta^{\dagger} \eta+\left(\mu^{2}+\delta \mu_{\rho}^{2}\right) \rho^{\dagger} \rho \\
& +\left(\mu^{2}+\delta \mu_{\chi}^{2}\right) \chi^{\dagger} \chi+\ldots
\end{aligned}
$$

with

$$
\begin{aligned}
\delta \mu_{\eta}^{2} \approx & {\left[4 g^{2}+20 \lambda_{1}+12 \lambda_{2}\right.} \\
& \left.-6 \sum_{\alpha=1}^{3}\left(\left|y_{\alpha}^{u}\right|^{2}+\left|g_{1 \alpha}^{d}\right|^{2}+\left|g_{2 \alpha}^{d}\right|^{2}\right)\right]\left(\frac{\Lambda}{4 \pi}\right)^{2}, \\
\delta \mu_{\rho}^{2} \approx & {\left[4 g^{2}+3 g_{X}^{2}+20 \lambda_{1}+12 \lambda_{2}\right.} \\
& \left.-6 \sum_{\alpha=1}^{3}\left(\left|g_{1 \alpha}^{u}\right|^{2}+\left|g_{2 \alpha}^{u}\right|^{2}+\left|y_{\alpha}^{d}\right|^{2}\right)\right]\left(\frac{\Lambda}{4 \pi}\right)^{2}, \\
\delta \mu_{\chi}^{2} \approx & {\left[4 g^{2}+3 g_{X}^{2}+20 \lambda_{1}+12 \lambda_{2}\right.} \\
& \left.-2 \sum_{a=1}^{3}\left|g_{a a}^{E}\right|^{2}-6\left|y^{J}\right|^{2}-6 \sum_{i=1}^{2}\left|g_{i i}^{j}\right|^{2}\right]\left(\frac{\Lambda}{4 \pi}\right)^{2} .
\end{aligned}
$$

Observe that the global $S U(3)_{L} \otimes S U(3)_{R}$ is recovered when $g_{X}$, and the Yukawa couplings in Eq. (7) are made equal to zero, resulting in $\delta \mu_{\eta}^{2}=\delta \mu_{\rho}^{2}=\delta \mu_{\chi}^{2}$, as it should be.

For the potential in Eq. (6) we have to have $v_{\eta}=v_{\rho}=$ $v_{\chi}$. The quantum corrections bring into the constraint equations, Eqs. (11) below, the effects of the explicit breakdown of $S U(3)_{L} \otimes S U(3)_{R}$, making it possible to have $v_{\eta} \neq v_{\rho} \neq v_{\chi}$. Considering the dominant contributions in Eqs. (10) and disregarding the corrections for the couplings in the self-interaction terms, the constraint equations for minimizing the potential are

$$
\begin{aligned}
& -\left(\mu^{2}+\delta \mu_{\eta}^{2}\right)=\lambda_{2} v_{\eta}^{2}+\lambda_{1} v^{2}+\frac{\sqrt{2}}{4} \frac{f}{v_{\eta}} v_{\rho} v_{\chi}, \\
& -\left(\mu^{2}+\delta \mu_{\rho}^{2}\right)=\lambda_{2} v_{\rho}^{2}+\lambda_{1} v^{2}+\frac{\sqrt{2}}{4} \frac{f}{v_{\rho}} v_{\eta} v_{\chi}, \\
& -\left(\mu^{2}+\delta \mu_{\chi}^{2}\right)=\lambda_{2} v_{\chi}^{2}+\lambda_{1} v^{2}+\frac{\sqrt{2}}{4} \frac{f}{v_{\chi}} v_{\rho} v_{\eta},
\end{aligned}
$$

where $v^{2}=v_{\eta}^{2}+v_{\rho}^{2}+v_{\chi}^{2}$. Since $\delta \mu_{\eta}^{2} \neq \delta \mu_{\rho}^{2} \neq \delta \mu_{\chi}^{2}$ it is possible to have $v_{\eta} \neq v_{\rho} \neq v_{\chi}$. Once the scale $\Lambda$ is really limited in the model, we do not expect that any severe fine-tuning is needed in Eqs. (11).

Next we show the masses, at leading order, for some of the scalar fields. There are two single charged scalars with masses given by

$$
\begin{aligned}
& M_{1+}^{2}=\left(\lambda_{2}-\frac{\sqrt{2}}{4} \frac{f v_{\chi}}{v_{\eta} v_{\rho}}\right)\left(v_{\eta}^{2}+v_{\rho}^{2}\right), \\
& M_{2+}^{2}=\left(\lambda_{2}-\frac{\sqrt{2}}{4} \frac{f v_{\rho}}{v_{\eta} v_{\chi}}\right)\left(v_{\eta}^{2}+v_{\chi}^{2}\right) ;
\end{aligned}
$$

a double charged scalar with mass given by

$$
M_{++}^{2}=\left(\lambda_{2}-\frac{\sqrt{2}}{4} \frac{f v_{\eta}}{v_{\chi} v_{\rho}}\right)\left(v_{\rho}^{2}+v_{\chi}^{2}\right)
$$

and a pseudoscalar ( $C P$ odd) with mass given by

$$
M_{A}^{2}=-\frac{\sqrt{2}}{4} f v_{\eta} v_{\rho} v_{\chi}\left(\frac{1}{v_{\chi}^{2}}+\frac{1}{v_{\rho}^{2}}+\frac{1}{v_{\eta}^{2}}\right) .
$$

Assuming $v_{\eta}, v_{\rho}$, and $v_{\chi}$ are real and positive, we have that $f<0$. This condition, along with $\lambda_{2}>0$, guarantees positive squared masses also for the charged scalars, as we see from Eqs. (12) and (13). We have not displayed the expressions for the last three $C P$ even scalars because they do not have a simple closed form.

The existence of an ultraviolet singularity in one of the running coupling constants, through a Landau-like pole, may indicate an energy scale at which new phenomena could intervene. It is not clear at all what new phenomena would arise at energies near or above the Landau-like pole in these 3-3-1 models, but it could modify the running of the low energy coupling constant. One possibility is the appearance of new particles from fields forming representations affecting the running of the coupling constants such that the pole is avoided at reachable energies [16]. An investigation with this hypothesis is needed in order to see how the electroweak scale would then be stabilized in this case.

Let us compare this sort of 3-3-1 model with the SUSY, LH, and ED solutions for the stabilization of the electroweak scale. SUSY, at the electroweak scale, is a renormalizable theory; however, we need to assume that the scale related with SUSY and the masses of the supersymmetric partners are at the $\mathrm{TeV}$ scale. It is also useful for the unification of three of the fundamental forces, but it has trouble with the stabilization of the proton [25]. The LH models are nonlinear realizations of the spontaneous symmetry breaking of a global symmetry, thus they are nonrenormalizable theories and remain perturbative, by construction, until an energy scale of the order of $10 \mathrm{TeV}$; to have naturalness beyond this scale, some similar mechanism has to be invoked, i.e., a second LH model, and so on. The ED proposals are also nonrenormalizable theories, and the energy scale to solve the hierarchy problem is chosen by hand as well. The 3-3-1 models are different in the following sense: they are renormalizable theories and the energy scale at the $\mathrm{TeV}$ scale is an intrinsic property of the theories. We did not assume that they have an appropriate value for solving the problem of the electroweak scale. The LH and ED solutions need ultraviolet completion; the 3-3-1 models do not. LH and ED solutions to the stabilization of the electroweak scale are $a d$ hoc since they are proposed just to solve the problem. The 3-3-1 models were proposed for other reasons and have interesting consequences and predictive power [24]. 
Finally, we stress that the argument that the running of $\sin ^{2} \theta_{W}$ gives an energy bound defining the model symmetry structure, as in Eq. (2), must be seen as a real prediction of this minimal 3-3-1 model. It is not possible to arbitrarily raise the scale related to the new particles in this model, which become inconsistent for energies $\mu$ at the standard model level such that $\sin ^{2} \theta_{W}(\mu)>0.25$. This gives us a reason why $\sin ^{2} \theta_{W}\left(M_{Z}\right)<0.25$ and also why there are no threatening divergences for the mass of the Higgs boson.

We have discussed the stabilization of the electroweak scale in a specific 3-3-1 model [11]. The same arguments we present here are valid for the version of the model in Ref. [12] which does not have heavy charged leptons, and a scalar sextet is mandatory for generating mass to the known charged leptons. It may be that there is a whole class of models where the arguments we have put forward here are valid.

A. G.D. thanks FAPESP for financial support. Both authors are supported partially by $\mathrm{CNPq}$ under the processes 302045/2007-4 (A.G.D.), 2007/04825-3, and 302102/2008-6 (V. P.).

\section{APPENDIX: MASS MATRICES}

\section{Scalar fields}

Defining $X=(1 / \sqrt{2})\left(v_{x}+X_{R}+i X_{I}\right)$, where $X=\eta$, $\rho, \chi$, we have for the mass matrix of the neutral real scalars, in the basis $\left(\eta_{R} \rho_{R} \chi_{R}\right)$,

$$
\mathbf{M}_{R}^{2}=\mu^{2} \mathbf{1}+\left[\begin{array}{ccc}
3 \lambda_{1} v_{\eta}^{2}+\lambda_{2}\left(2 v_{\eta}^{2}+v^{2}\right) & 2 \lambda_{1} v_{\eta} v_{\rho}+\frac{\sqrt{2}}{4} f v_{\chi} & 2 \lambda_{1} v_{\eta} v_{\chi}+\frac{\sqrt{2}}{4} f v_{\rho} \\
2 \lambda_{1} v_{\eta} v_{\rho}+\frac{\sqrt{2}}{4} f v_{\chi} & 3 \lambda_{1} v_{\rho}^{2}+\lambda_{2}\left(2 v_{\rho}^{2}+v^{2}\right) & 2 \lambda_{1} v_{\rho} v_{\chi}+\frac{\sqrt{2}}{4} f v_{\eta} \\
2 \lambda_{1} v_{\eta} v_{\chi}+\frac{\sqrt{2}}{4} f v_{\rho} & 2 \lambda_{1} v_{\rho} v_{\chi}+\frac{\sqrt{2}}{4} f v_{\eta} & 3 \lambda_{1} v_{\chi}^{2}+\lambda_{2}\left(2 v_{\chi}^{2}+v^{2}\right)
\end{array}\right]
$$

for the neutral pseudoscalars, in the basis $\left(\eta_{I} \rho_{I} \chi_{I}\right)$,

$$
\mathbf{M}_{I}^{2}=\mu^{2} \mathbf{1}+\left[\begin{array}{ccc}
\lambda_{1} v^{2}+\lambda_{2} v_{\eta}^{2} & -\frac{\sqrt{2}}{4} f v_{\chi} & -\frac{\sqrt{2}}{4} f v_{\rho} \\
-\frac{\sqrt{2}}{4} f v_{\chi} & \mu^{2}+\lambda_{1} v^{2}+\lambda_{2} v_{\rho}^{2} & -\frac{\sqrt{2}}{4} f v_{\eta} \\
-\frac{\sqrt{2}}{4} f v_{\rho} & -\frac{\sqrt{2}}{4} f v_{\eta} & \lambda_{1} v^{2}+\lambda_{2} v_{\chi}^{2}
\end{array}\right]
$$

for the single charged scalars, in the basis $\left(\eta_{1}^{-} \rho^{-}\right)$,

$$
\mathbf{M}_{1+}^{2}=\mu^{2} \mathbf{1}+\left[\begin{array}{cc}
\lambda_{1} v^{2}+\lambda_{2}\left(v^{2}-v_{\chi}^{2}\right) & \lambda_{2} v_{\eta} v_{\rho}-\frac{\sqrt{2}}{4} f v_{\chi} \\
\lambda_{2} v_{\eta} v_{\rho}-\frac{\sqrt{2}}{4} f v_{\chi} & \lambda_{1} v^{2}+\lambda_{2}\left(v^{2}-v_{\chi}^{2}\right)
\end{array}\right],
$$

and, in the $\left(\eta_{2}^{-} \chi^{-}\right)$basis,

$$
\mathbf{M}_{2+}^{2}=\mu^{2} \mathbf{1}+\left[\begin{array}{cc}
\lambda_{1} v^{2}+\lambda_{2}\left(v^{2}-v_{\rho}^{2}\right) & \lambda_{2} v_{\eta} v_{\chi}-\frac{\sqrt{2}}{4} f v_{\rho} \\
\lambda_{2} v_{\eta} v_{\chi}-\frac{\sqrt{2}}{4} f v_{\rho} & \lambda_{1} v^{2}+\lambda_{2}\left(v^{2}-v_{\rho}^{2}\right)
\end{array}\right]
$$

and for the double charged scalars, in the basis $\left(\rho^{--} \chi^{--}\right)$,

$$
\mathbf{M}_{++}^{2}=\mu^{2} \mathbf{1}+\left[\begin{array}{cc}
\lambda_{1} v^{2}+\lambda_{2}\left(v^{2}-v_{\eta}^{2}\right) & \lambda_{2} v_{\rho} v_{\chi}-\frac{\sqrt{2}}{4} f v_{\eta} \\
\lambda_{2} v_{\rho} v_{\chi}-\frac{\sqrt{2}}{4} f v_{\eta} & \lambda_{1} v^{2}+\lambda_{2}\left(v^{2}-v_{\eta}^{2}\right)
\end{array}\right]
$$

\section{Gauge bosons}

For the real neutral gauge bosons in the $\left(W_{\mu}^{3}, W_{\mu}^{8}, B_{\mu}\right)$ basis, the mass matrix is

$$
M_{\text {n.g.b. }}^{2}=\left(\begin{array}{lcc}
\frac{g^{2}}{4}\left(v_{\eta}^{2}+v_{\rho}^{2}\right) & \frac{\sqrt{3} g^{2}}{12}\left(v_{\eta}^{2}-v_{\rho}^{2}\right) & -\frac{g_{X} g}{2} v_{\rho}^{2} \\
\frac{\sqrt{3} g^{2}}{12}\left(v_{\eta}^{2}-v_{\rho}^{2}\right) & \frac{g^{2}}{12}\left(v^{2}+3 v_{\chi}^{2}\right) & \frac{\sqrt{3}}{6} g_{X} g\left(v_{\rho}^{2}+2 v_{\chi}^{2}\right) \\
\frac{g^{2}}{12}\left(v^{2}+3 v_{\chi}^{2}\right) & \frac{\sqrt{3}}{6} g_{X} g\left(v_{\rho}^{2}+2 v_{\chi}^{2}\right) & g_{X}^{2}\left(v_{\rho}^{2}+v_{\chi}^{2}\right)
\end{array}\right) ;
$$

and for the non-Hermitian gauge bosons

$$
M_{W}^{2}=\frac{g^{2}}{4}\left(v_{\eta}^{2}+v_{\rho}^{2}\right), \quad M_{V}^{2}=\frac{g^{2}}{4}\left(v_{\eta}^{2}+v_{\chi}^{2}\right), \quad M_{U}^{2}=\frac{g^{2}}{4}\left(v_{\rho}^{2}+v_{\chi}^{2}\right) .
$$




\section{Fermion fields}

For type $u$ and $d$ quarks

$$
M_{u}=\frac{1}{\sqrt{2}}\left(\begin{array}{ccc}
g_{11}^{u} v_{\rho}^{*} & g_{12}^{u} v_{\rho}^{*} & g_{13}^{u} v_{\rho}^{*} \\
g_{21}^{u} v_{\rho}^{*} & g_{22}^{u} v_{\rho}^{*} & g_{23}^{u} v_{\rho}^{*} \\
y_{1}^{u} v_{\eta} & y_{2}^{u} v_{\eta} & y_{3}^{u} v_{\eta}
\end{array}\right), \quad M_{d}=\frac{1}{\sqrt{2}}\left(\begin{array}{cccc}
g_{11}^{d} v_{\eta}^{*} & g_{12}^{d} v_{\eta}^{*} & g_{13}^{d} v_{\eta}^{*} \\
g_{21}^{d} v_{\eta}^{*} & g_{22}^{d} v_{\eta}^{*} & g_{23}^{d} v_{\eta}^{*} \\
y_{1}^{d} v_{\rho} & y_{2}^{d} v_{\rho} & y_{3}^{d} v_{\rho}
\end{array}\right)
$$

for the $j_{i}$ and $J$ quarks, and exotic leptons

$$
M_{j}=\left(\begin{array}{ll}
g_{11}^{j} & g_{12}^{j} \\
g_{21}^{j} & g_{22}^{j}
\end{array}\right) \frac{v_{\chi}}{\sqrt{2}}, \quad M_{J}=\frac{y^{J}}{\sqrt{2}} v_{\chi} ; \quad M_{E}=\left(\begin{array}{lll}
g_{11}^{E} & g_{12}^{E} & g_{13}^{E} \\
g_{21}^{E} & g_{22}^{E} & g_{23}^{E} \\
g_{31}^{E} & g_{32}^{E} & g_{33}^{E}
\end{array}\right) \frac{v_{\chi}}{\sqrt{2}} .
$$

[1] The cutoff $\Lambda$ is used here for evaluating the momentum integrals and is related to new physics. There have been interesting developments concerning conformal symmetry, where dimensional regularization is argued to be more appropriated, since it violates this symmetry in a mild way when compared with the cutoff method. See K. A. Meissner and H. Nicolai, Phys. Lett. B 648, 312 (2007).

[2] M. Veltman, Acta Phys. Pol. B 12, 437 (1981).

[3] C. Amsler et al., Phys. Lett. B 667, 1 (2008).

[4] LEP and SLD Electroweak Working Group, Phys. Rep. 427, 257 (2006).

[5] R. K. Kaul, Phys. Lett. 109B, 19 (1982).

[6] N. Arkani-Hamed, A. G. Cohen, and H. Georgi, Phys. Lett. B 513, 232 (2001); N. Arkani-Hamed, A. G. Cohen, E. Katz, and A.E. Nelson, J. High Energy Phys. 07 (2002) 034.

[7] M. Schmaltz and D. Tucker-Smith, Annu. Rev. Nucl. Part. Sci. 55, 229 (2005).

[8] Y. Hosotani, Phys. Rev. D 29, 731 (1984); L. Randall and R. Sundrum, Phys. Rev. Lett. 83, 3370 (1999).

[9] M. Schmaltz, J. High Energy Phys. 08 (2004) 056; O. C. W. Kong, J. Korean Phys. Soc. 45, S404 (2004).

[10] J. C. Montero, F. Pisano, and V. Pleitez, Phys. Rev. D 47, 2918 (1993); R. Foot, H. N. Long, and T. A. Tran, Phys. Rev. D 50, R34 (1994); H. N. Long, Phys. Rev. D 53, 437 (1996).
[11] F. Pisano and V. Pleitez, Phys. Rev. D 46, 410 (1992); P. H. Frampton, Phys. Rev. Lett. 69, 2889 (1992).

[12] V. Pleitez and M. Tonasse, Phys. Rev. D 48, 2353 (1993).

[13] P. H. Frampton, Mod. Phys. Lett. A 18, 1377 (2003).

[14] P. Das and P. Jain, Phys. Rev. D 62, 075001 (2000).

[15] A. G. Dias, R. Martinez, and V. Pleitez, Eur. Phys. J. C 39, 101 (2005).

[16] A. G. Dias, Phys. Rev. D 71, 015009 (2005); R. Martinez and F. Ochoa, Eur. Phys. J. C 51, 701 (2007).

[17] E. R. Barreto, Y. A. Coutinho, and J. S. Borges, Eur. Phys. J. C 50, 909 (2007).

[18] M. B. Tully and G. C. Joshi, Phys. Lett. B 466, 333 (1999).

[19] E. R. Barreto, Y.A. Coutinho, and J.S. Borges, Nucl. Phys. B810, 210 (2009).

[20] F. Ramirez-Zavaleta, G. Tavares-Velasco, and J. J. Toscano, Phys. Rev. D 75, 075008 (2007); A. FloresTlalpa, J. Montano, F. Ramirez-Zavaleta, and J.J. Toscano, Phys. Rev. D 80, 033006 (2009); B. Meirose and A. J. Ramalho, J. Phys. G 36, 095007 (2009).

[21] S. Coleman and E. Weinberg, Phys. Rev. D 7, 1888 (1973).

[22] M. Sher, Phys. Rep. 179, 273 (1989).

[23] R. Jackiw, Phys. Rev. D 9, 1686 (1974).

[24] A. G. Dias, J.C. Montero, and V. Pleitez, Phys. Lett. B 637, 85 (2006); Phys. Rev. D 73, 113004 (2006).

[25] H. Murayama and A. Pierce, Phys. Rev. D 65, 055009 (2002). 